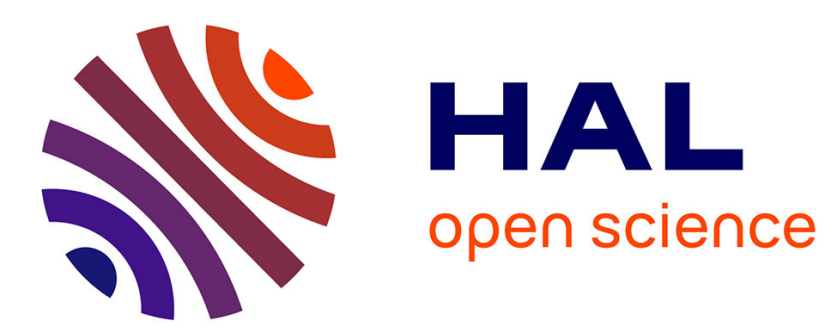

\title{
FES-Induced Torque Prediction with Evoked EMG Sensing for Muscle Fatigue Tracking
} Qin Zhang, Mitsuhiro Hayashibe, Philippe Fraisse, David Guiraud

\section{To cite this version:}

Qin Zhang, Mitsuhiro Hayashibe, Philippe Fraisse, David Guiraud. FES-Induced Torque Prediction with Evoked EMG Sensing for Muscle Fatigue Tracking. IEEE/ASME Transactions on Mechatronics, 2011, 16(5), pp.816 - 826. 10.1109/TMECH.2011.2160809 . lirmm-00604670

\section{HAL Id: lirmm-00604670 https://hal-lirmm.ccsd.cnrs.fr/lirmm-00604670}

Submitted on 29 Jun 2011

HAL is a multi-disciplinary open access archive for the deposit and dissemination of scientific research documents, whether they are published or not. The documents may come from teaching and research institutions in France or abroad, or from public or private research centers.
L'archive ouverte pluridisciplinaire HAL, est destinée au dépôt et à la diffusion de documents scientifiques de niveau recherche, publiés ou non, émanant des établissements d'enseignement et de recherche français ou étrangers, des laboratoires publics ou privés. 


\title{
FES-Induced Torque Prediction with Evoked EMG Sensing for Muscle Fatigue Tracking
}

\author{
Qin Zhang, Student Member, IEEE, Mitsuhiro Hayashibe, Member, IEEE, Philippe Fraisse, Member, IEEE, \\ David Guiraud, Member, IEEE
}

\begin{abstract}
This study investigates a torque estimation method for muscle fatigue tracking, using stimulus evoked electromyography (eEMG) in the context of a functional electrical stimulation (FES) rehabilitation system. Although FES is able to effectively restore motor function in spinal cord injured (SCI) individuals, its application is inevitably restricted by muscle fatigue. In addition, the sensory feedback indicating fatigue is missing in such patients. Therefore, torque estimation is essential to provide feedback or feedforward signal for adaptive FES control. In this work, a fatigue-inducing protocol is conducted on five SCI subjects via transcutaneous electrodes under isometric condition, and eEMG signals are collected by surface electrodes. A myoelectrical mechanical muscle model based on the Hammerstein structure with eEMG as model input is employed to capture muscle contraction dynamics. It is demonstrated that the correlation between eEMG and torque is time-varying during muscle fatigue. Compared to conventional fixed-parameter models, the adaptedparameter model shows better torque prediction performance in fatiguing muscles. It motivates us to use a Kalman filter with forgetting factor for estimating the time-varying parameters and for tracking muscle fatigue. The assessment with experimental data reveals that the identified eEMG-to-torque model properly predicts fatiguing muscle behavior. Furthermore, the performance of the time-varying parameter estimation is efficient, suggesting that real-time tracking is feasible with a Kalman filter and driven by eEMG sensing in the application of FES.
\end{abstract}

Index Terms-Evoked Electromyography (eEMG), Torque Prediction, Muscle Fatigue Tracking, Kalman Filter with Forgetting Factor, Functional Electrical Stimulation (FES).

\section{INTRODUCTION}

\section{A. Background}

$\mathbf{F}$ UNCTIONAL electrical stimulation (FES) is one of the existing solutions to partly restore lost motor function in persons with spinal cord injury (SCI). The electrical stimulus can artificially generate action potential on the axons of the alpha motor neurons to drive muscle contraction in place of the central nervous system (CNS). FES has been used in a wide range of rehabilitation applications, including the FES-aided support of standing, gait, grasping [1] [2], drop foot correction, tremor compensation and bladder/bowel management.

Although FES has potential advantages for the improvement of functional restoration in SCI subjects, it has not so far gained widespread clinical use because of its limitations, such

This paper was presented in part at International Conference of the IEEE Engineering in Medicine and Biology Society (EMBS), Buenos Aires, Argentina, August 31- September 4, 2010.

The authors are with DEMAR Project, INRIA Sophia-Antipolis and LIRMM, UMR5506 CNRS UM2, 161 Rue Ada, 34095 Montpellier, Cedex 5, France. \{qin.zhang, hayashibe, fraisse, guiraud\}@lirmm.fr as rapid muscle fatigue and imprecise force/torque control. In order to obtain the desired movement or trajectory for a certain task, the electrical stimulation (ES) must produce strong, consistent muscle force. It is well known, however, that the fatigue resistance decreases in the paralyzed muscle after SCI [3], so that muscle becomes fatigued more rapidly when artificially stimulated than when excited by the CNS, because of the way that the motor units are recruited: inverse size principle, synchronized activation of motor units and constant order of recruitment [4]. In addition, since SCI individuals have also lost their sensory pathways, they cannot perceive muscle fatigue as stimulation proceeds, which leads to movement failures and sub-optimal FES parameter-tuning in practical FES control. Furthermore, the physiological and mechanical complexity - and nonlinearity — of the neuromuscular system increase the difficulty for FES to precisely control muscle force/torque output and to perform functional movements [5].

Some research has addressed the attenuation or delay of FES-induced fatigue. Optimal stimulation patterns, such as Nlets [6] or catch-like stimulation [7], were found to maximize muscle performance and minimize fatigue. Random modulation of FES parameters was proved to have no effect on muscle fatigue [8]. Low frequency and long pulse duration was reported to be capable of producing less fatigue with constant frequency and intensity [9]. Despite these efforts, no consensus was reached to date due to the complex, multifactorial and task-specific properties of muscle fatigue [10]. Therefore, muscle fatigue is still a major limiting factor for the widespread application of FES.

Accurate torque/force signal is hence important to produce the desired feedback or feedforward signal for adaptive FES control in the the presence of muscle fatigue. However, the torque/force measurement via external equipments is not convenient for daily use, and generally do not directly measure the muscle output induced by stimulation. The implanted sensor is one possible technique [11] but is not yet available for practical use. Thus, the inadequacy of torque/force sensors is another problem in rehabilitation application. This motivates the development of methods to estimate muscle torque from biosignals that can be measured or well estimated.

\section{B. Related Work}

As described above, on one hand, muscle fatigue may result in torque/force decline and eventually movement failure without any sensation in SCI individuals, on the other hand, convenient and precise sensor is not available for measuring 
muscle output generated by stimulation. Therefore, estimating the FES-induced torque/force is important for adaptive closedloop FES control, considering the compensation of fatigue. Several techniques have been developed for force/torque prediction in fatiguing muscle. Some mathematical fatigue models were developed from physiological knowledge or experimental analysis. A fatigue function was introduced into a biomechanical model to predict FES-induced shank motion [12]. A fatigue recovery function, based on metabolic profiles, was introduced into a musculotendon model [13]. A four-parameter fatigue model, coupled with a mathematic isometric force model, that predicts the fatigue induced by different stimulation patterns under isometric contractions, was reported in [14]. However, in the above works, the complex model parameters remain difficult to be identified in application, because of high nonlinearity and dynamic complexities. Moreover, the fatigue models can work only when the stimulation scheme can be predetermined. But in practice, the stimulation pattern is unknown in advance, so the fatigue model cannot work.

Some researchers proposed to use FES-evoked electromyography (eEMG) for torque/force prediction, as ES-induced muscle contraction represents both electrical and mechanical behavior, respectively manifested by eEMG and torque/force. Moreover, the eEMG signal permits noninvasive and reliable measurement of muscle activity. Under continuous stimulation, eEMG was found to be highly correlated with FES-induced torque in different muscle conditions, suggesting its use as a fatigue indicator [15] [16]. An exponential function was used to express the electrical and mechanical relationship of the stimulated muscle in SCI patients [17] [18]. A predictive model based on eEMG instead of stimulation was developed, allowing the use of eEMG as a synthetic torque sensor [19]. One possible explanation is that eEMG can capture most time-variations due to the effects of fatigue, which cannot be captured by a stimulation-to-torque model. Combining timedomain and frequency-domain variables was suggested for better prediction of force [18]. In these works, a fixed relationship between eEMG and muscle force/torque during sustained stimulation was assumed. Nevertheless, this correlation was presented to be time-variant during different muscle-fatigue levels [20] and during recovery process [21]. Such evidence indicates that the relationship between eEMG and muscle force/torque is not constant but time-varying, and also implies the limitations of previous force/torque prediction strategies based on fixed-parameter models. [21] suggested an adaptive tuning of the parameters of eEMG for predicting the stimulated force. However, they did not propose a feasible and effective method to do this.

The present work aims to develop an online estimation method of FES-induced torque based on eEMG in the presence of muscle fatigue. This paper is organized as follows. The relevant experiment is introduced in Section II. The muscle modeling is presented in Section III. The proposed estimation method, Kalman filter with forgetting factor, is presented in Section IV. In Section V, we show the identification and prediction results with the ordinary least squares method and the Kalman filter technique. Discussions and conclusions can be found in Sections VI and VII, respectively.

\section{EXPERIMENTAL METHODS}

\section{A. Experimental set-up}

The experiments were conducted on five SCI subjects (see TABLE I) in the PROPARA rehabilitation center, Montpellier, France. All subjects were classified as the American Spinal Injury Association (ASIA) A, where no motor or sensory function is preserved. The experimental set-up is depicted in Fig. 1. This study was approved by the ethical committee of France and all subjects signed informed consent forms.

TABLE I

PATIENT CONFIGURATIONS

\begin{tabular}{c|c|c|c|c|c}
\hline $\begin{array}{c}\text { Test } \\
\text { Subject }\end{array}$ & $\begin{array}{c}\text { Age } \\
\text { (years) }\end{array}$ & $\begin{array}{c}\text { Weight } \\
(\mathrm{kg})\end{array}$ & $\begin{array}{c}\text { Height } \\
(\mathrm{cm})\end{array}$ & $\begin{array}{c}\text { Level } \\
\text { of injury* }\end{array}$ & $\begin{array}{c}\text { Months } \\
\text { post injury }\end{array}$ \\
\hline S1 & 39 & 50 & 169 & T6 & 3 \\
\hline S2 & 22 & 54 & 172 & C7 & 30 \\
\hline S3 & 26 & 64 & 192 & T6 & 36 \\
\hline S4 & 32 & 61.5 & 177 & C5 & 8 \\
\hline S5 & 48 & 76 & 177 & T6 & 18 \\
\hline
\end{tabular}

* Level of injury corresponds to the injured location in vertebral segments T6 means the 6th thoracic (chest) vertebra while $\mathrm{C}$ represents cervical (neck) vertebra.

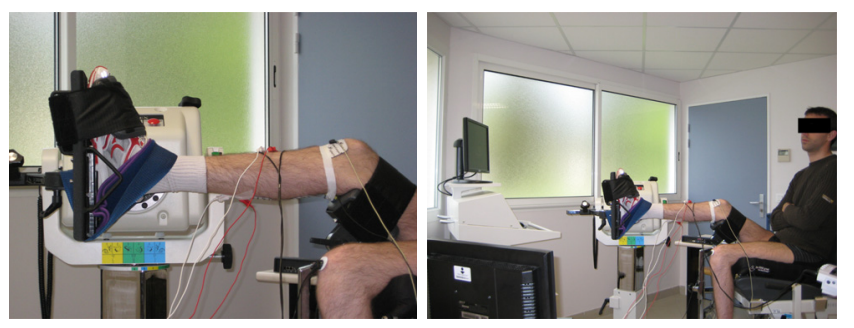

Fig. 1. Experimental set-up for electrical stimulation and ankle torque measurement.

The subjects were seated on the chair with the ankle at $90^{\circ}$, while the joint center was aligned with the axis of a calibrated dynamometer (Biodex 3, Shirley corp., NY, USA). The shank was adjusted to be horizontal to the ground with the knee joint at $40^{\circ}$. The foot was strapped to the pedal to transmit ankle torque to the dynamometer and to allow the optimal recording of isometric ankle torque. Electrical current pulses were delivered to the right triceps surae muscle group via surface electrodes $(10 \mathrm{~cm} \times 3 \mathrm{~cm})$ to induce muscle contractions and to plantarflex the ankle joint. One electrode was placed $5 \mathrm{~cm}$ beneath the popliteal cavity and the other beneath the insertion point of the medial and lateral gastrocnemius on the Achilles tendon. The muscle group was stimulated with amplitude modulation at a constant frequency $(30 \mathrm{~Hz})$ and constant pulse-width $(450 \mu \mathrm{s})$, under isometric conditions, by a portable stimulator (Cefar physio 4, Cefar Medical, Lund, Sweden).

EEMG activity of soleus in triceps surae muscle group was recorded, amplified (gain 1000) and sampled at $4 \mathrm{KHz}$ by an acquisition system (Biopac MP100, Biopac Systems Inc., Santa Barbara, CA, USA). The bipolar AgCl EMG electrodes were positioned over the muscle belly in the direction of muscle fiber with $20 \mathrm{~mm}$ interelectrode spacing. The reference electrode was placed on the patella of another leg. The skin 
under the electrodes was shaved to minimize the impedance. Isometric ankle plantar-flexion torque was measured using the dynamometer (Biodex 3), sampled at $2 \mathrm{KHz}$, and interfaced with the acquisition system (Biopac MP100).

\section{B. Experimental Protocol}

For each subject, the experiment consists of three test sessions: a fatigue-inducing test, a fatigue-recovery test and a random test, as shown in Fig. 2. The maximum stimulation amplitude was found for each subject at the beginning, by gradually increasing stimulation amplitude until torque saturation. The fatigue-inducing test includes several sequences (named as fatigue1-fatigue5). Each sequence contains five trapezoidal trains with each trapezoidal train consisting of $4 \mathrm{~s}$ stimulation (1s ramp-up, 2s plateau and 1s ramp-down) and $2 \mathrm{~s}$ rest. The stimulation amplitude during plateau is chosen at $50 \%$ of the maximum stimulation amplitude with constant stimulation frequency $30 \mathrm{~Hz}$ and constant stimulation pulse width $450 \mu \mathrm{s}$. In order to induce muscle fatigue, three such stimulation sequences were applied to subjects S1 and S2, four sequences to S3, and five sequences to S4 and S5. In fatigue-recovery test, one trapezoidal train (indicated by pre, postA, ..., postE and recovery $1, \ldots$ recovery 3 ) at maximum stimulation amplitude was delivered to the muscle just before and after each fatigue-inducing sequence. After the stimulation train postE, the same stimulation train was applied every 5 minutes, up to 15 minutes. At the end, a sequence including several trapezoidal trains was applied. In this case, the stimulation amplitude in each train was increased from zero to a randomly determined value below the maximum value, and then symmetrically decreased, during two minutes in total.

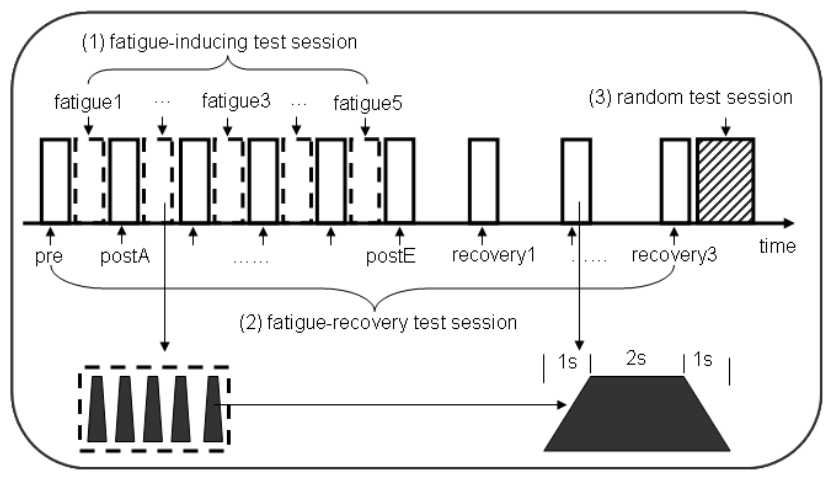

Fig. 2. Schematic representation of the experimental sessions.

\section{Torque and eEMG Data Processing}

During electrically elicited contractions, the detected surface eEMG signal is contaminated by stimulation artifacts due to the stimulation current which is recorded by the eEMG amplifier. For the preparation of model identification and torque prediction based on eEMG, the measured eEMG and torque were processed in the following steps. An example of the processed results is illustrated in Fig. 3.

1) The blanking window method [22] is used to remove stimulation artifacts from the raw eEMG signal and extract muscle response (Mwave).
2) A lowpass filter is applied to measured ankle torque (6thorder, cutoff frequency $100 \mathrm{~Hz}$ ) and measured eEMG (6th-order, cutoff frequency $300 \mathrm{~Hz}$ ).

3) The filtered eEMG signal is divided into epochs with each epoch containing one Mwave, and the mean absolute value (MAV) of eEMG is calculated every five epochs. The average torque is calculated within the same time window.

4) The MAV and average torque are normalized with respect to their maximum values.

The normalized MAV and normalized torque are prepared to be the system input and output for model identification.
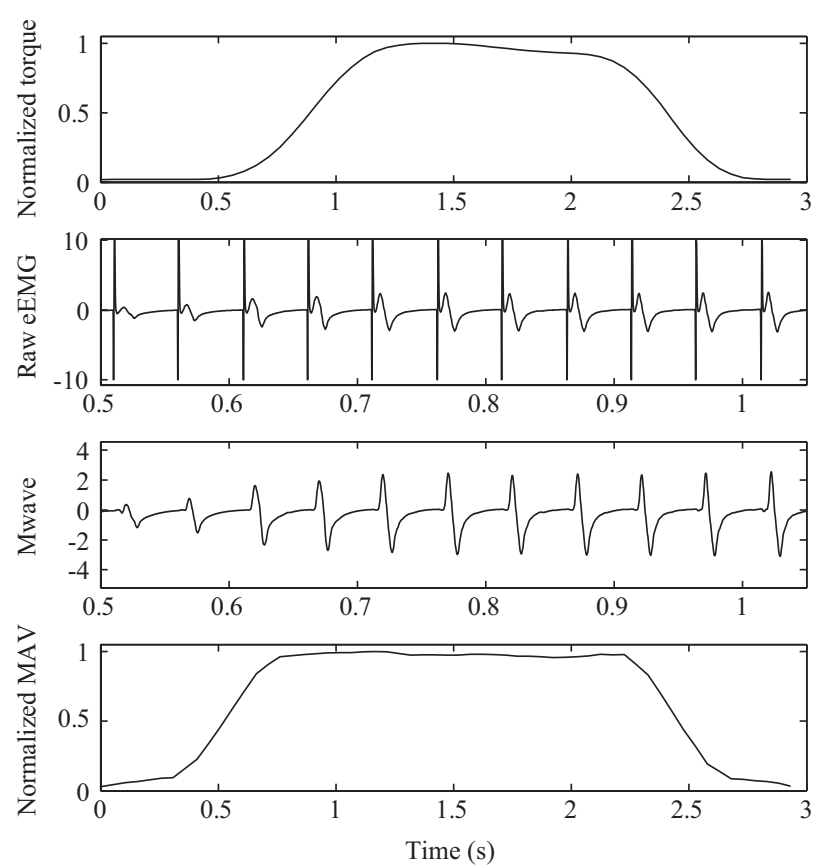

Fig. 3. An example of processed eEMG and torque. The raw eEMG signal and Mwave were zoomed in $(0.5 s \sim 1.1 s)$ to show the details during stimulation increase. The raw eEMG was contaminated by stimulation artifacts. Blanking window was applied to remove artifacts so that Mwave was effectively extracted.

\section{CONTRACTION DYNAMICS MODEL OF ELECTRICALLY STIMULATED MUSCLE}

\section{A. Model Structure}

The discrete-time Hammerstein structure was used to model the muscle contraction dynamics as shown in Fig. 4. This model consists of a memoryless nonlinear part followed by a dynamic linear part. It is popularly used to represent highly nonlinear systems, and particularly useful for modeling biomechanical systems, such as stretch reflex EMG signal [23] and electrically-stimulated muscles, relating stimulation to muscle force under isometric conditions [24]. It has been shown to extend to dynamic conditions [25], which is essential for developing stable adaptive controllers for applications in FES. In this study, the MAV of eEMG and FES-induced ankle torque are system input $u(t)$ and output $y(t)$, respectively. 


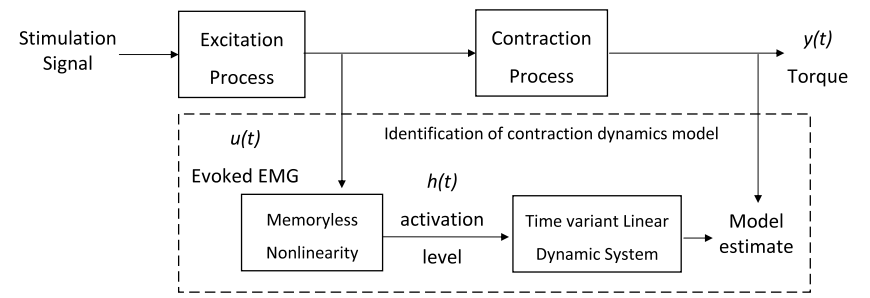

Fig. 4. Muscle model structure. The present work focuses on the contraction dynamics model contained in the rectangle within the dashed line. The memoryless nonlinearity is modeled by an $n$ th-order polynomial function. The linear dynamics is chosen as an ARX model.

The memoryless nonlinear function maps the system input $u(t)$, to the intermediate variable $h(t)$, which represents the activation level of the stimulated muscle. It is traditionally modeled by an $n$ th-order polynomial of $u(t)$ [23] as follows:

$$
h(t)=\sum_{i=1}^{n} \gamma_{i} u^{i}(t)
$$

where $\gamma_{i}$ is a model coefficient.

The linear time-variant system is described by an autoregressive model with exogenous input (ARX), which has been shown experimentally to yield good prediction of output torque/force in isometric situation [26]. It can be described as

$$
A(z) y(t)=B(z) h(t)+e(t)
$$

with transfer function $G(z)=B(z) / A(z)$ and

$$
\begin{aligned}
& A(z)=1+a_{1} z^{-1}+a_{2} z^{-2}+\cdots+a_{l} z^{-l} \\
& B(z)=b_{1} z^{-1}+b_{2} z^{-2}+\cdots+b_{m} z^{-m}
\end{aligned}
$$

where $z^{-1}$ is the backward shift operator which makes $z^{-1} y(t)=y(t-1)$. In (2), $y(t)$ is the system output at time $t, e(t)$ is zero mean and Gaussian white noise affecting the system. The $h(t)$ is the output of the nonlinear element, and the input of the linear element as seen in Fig. 4.

Substituting (1) and (3) into (2) and expanding, the output of a polynomial Hammerstein model (PHM) at a given time $t$ can be parameterized as:

$$
y(t, \theta)=\sum_{i=1}^{l} a_{i} y(t-i)+\sum_{i=1}^{m} \sum_{j=1}^{n} \mu_{i j}(u(t-i))^{j}
$$

where $\mu_{i j}=b_{i} \gamma_{j}, \theta=\left[a_{1}, \cdots, a_{l}, \mu_{11}, \cdots, \mu_{m n}\right]^{T}$ is a parameter vector containing the model coefficients. The size of $\theta$ depends on model complexity. Therefore, the selection of model order $(l, m, n)$ is a key step in the estimation of the unknown parameters in $\theta$. We chose to model the recruitment curve of the muscle as a 3rd-order polynomial of instantaneous MAV of eEMG, $(n=3)$, as in [24]. Linear model order determination was determined by comparing the Rissanen's minimum description length (MDL) [27][28] obtained for different model orders, since the MDL principle provides a criterion for tradeoff between the simplicity of the model and the model's applicability to the data. Model-order parameters ranging from $2 \leq l \leq 6$, and from $2 \leq m \leq 6$ were considered. Finally, model order $(l=3, m=4)$ was chosen with relatively less MDL value and a simpler model as shown in Fig. 5. The stimulated muscle model has $l+m \times n$ unknown parameters in all. The elements of $\theta$ as well as the eEMG-totorque features are time-varying due to the effects of fatigue and the associated biochemistry. At a given time $t$, the model estimates are predicted using (4) by assuming the system is stationary, or slowly time-varying, during the prediction horizon.

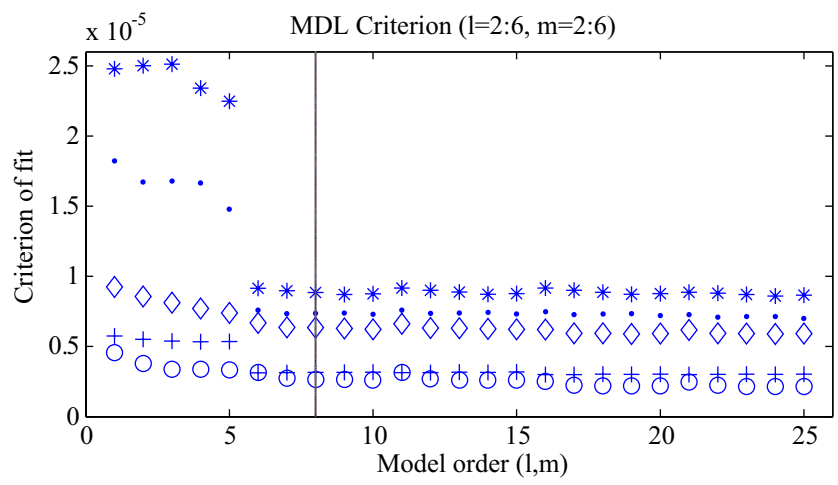

Fig. 5. Criterion of fit for all datasets of fatigue-inducing test in 5 subjects

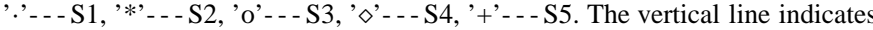
the selected model order $(3,4)$.

The computation of past torque $y(t-i)$ in (4) has two possible versions: (i) past measured torque $y_{m}(t-i)$ and (ii) past predicted torque $\widehat{y}_{p}(t-i)$. For model identification, the former approach is preferred, as measured torque is favorable for model fitting. For torque prediction, both can be employed, while from a practical point of view, the former is inappropriate, since measured torque is not available. Accordingly, the corresponding predicted output $\widehat{y}(t)$ based on identified model has two versions, $\widehat{y}_{m}(t)$ and $\widehat{y}_{p}(t)$, which can be computed as a function of past measured MAV of eEMG, past measured torque or past predicted torque in this way:

$$
\begin{aligned}
\widehat{y}_{m}(t)= & f u n c[u(t-1), u(t-2), \ldots u(t-m), \\
& \left.y_{m}(t-1), y_{m}(t-2), \ldots y_{m}(t-l)\right] \\
\widehat{y}_{p}(t)= & f u n c[u(t-1), u(t-2), \ldots u(t-m), \\
& \left.\widehat{y}_{p}(t-1), \widehat{y}_{p}(t-2), \ldots \widehat{y}_{p}(t-l)\right]
\end{aligned}
$$

When torque measurement is not available, the second approach as shown in (6) makes it possible to use eEMG as a synthetic torque sensor. In this case, we can initialize the predicted torque at zero when no stimulation is delivered to the muscle.

\section{B. State-Space Model Representation}

State-space form is basically required for the implementation of Kalman filter. Considering a PHM $(l, m, n)$ as in (4), its state-space form can be compactly written as:

1) process equation

$$
\mathbf{x}_{k}=\mathbf{f}\left(\mathbf{x}_{k-1}, u_{k-1}\right)=\mathbf{A} \mathbf{x}_{k-1}+\sum_{i=1}^{n} \mathbf{B}_{i}\left[u_{k-1}\right]^{i}
$$

2) measurement equation

$$
y_{k}=\mathbf{C x}_{k}
$$


where the subscript $k$ indicates the current time step. In (7), the current state vector $\mathbf{x}_{\mathbf{k}}=\left[x_{1, k}, x_{2, k}, \cdots, x_{q, k}\right]^{T}, q=$ $\max \{l, m\}$, and $u_{k-1}$ is the previous model input. $\mathbf{A} \in \mathbb{R}^{q \times q}$ relates the previous state $\mathbf{x}_{k-1}$ to the current state $\mathbf{x}_{k} . \mathbf{B}_{i} \in$ $\mathbb{R}^{q \times 1}$ relates the previous model input $\left[u_{k-1}\right]^{i}$ to the current state $\mathbf{x}_{k}$. They can be represented as following:

$$
\mathbf{A}=\left[\begin{array}{cccccc}
a_{1} & 1 & 0 & \cdots & 0 & 0 \\
a_{2} & 0 & 1 & \cdots & 0 & 0 \\
\vdots & \vdots & \vdots & \ddots & \vdots & \vdots \\
a_{q-1} & 0 & 0 & \ldots & 0 & 1 \\
a_{q} & 0 & 0 & \ldots & 0 & 0
\end{array}\right], \quad \mathbf{B}_{i}=\left[\begin{array}{c}
\mu_{1, i} \\
\mu_{2, i} \\
\vdots \\
\mu_{q-1, i} \\
\mu_{q, i}
\end{array}\right]
$$

The $y_{k}$ in (8) is the measurement of system output. $\mathbf{C} \in$ $\mathbb{R}^{1 \times q}$ relates the current state $\mathbf{x}_{k}$ to the current measurement $y_{k}$ with the following expression:

$$
\mathbf{C}=\left[\begin{array}{lllll}
1 & 0 & \cdots & 0 & 0
\end{array}\right]
$$

Note that, in practice matrices $\mathbf{A}, \mathbf{B}_{i}$ and $\mathbf{C}$ might change with each time step or measurement, but here we assume they are constant for simplifying the expression.

\section{IDENTIFICATION USING KALMAN FILTER WITH FORGETTING FACTOR}

Recursive estimates are important when parameter identification is needed in real-time, such as when the information is used in adaptive control or real-time diagnosis. The Kalman filter is an efficient recursive filter that estimates the internal states and parameters of a discrete-time system from a series of noisy measurements. Online estimation of the states in $\mathbf{x}$ and of the model parameters in $\theta$ is investigated simultaneously in this work. Model parameters to be identified are the coefficients relating to both the past measured torque and the past measured MAV of eEMG in (4). A Kalman filter for state and parameter estimation is performed by regarding the unknown model parameters as elements of the state vector. In this way, the basic Kalman filter algorithm does not need to be modified, except that the state vector $\mathbf{x}$ will be augmented with the unknown parameters in $\theta$. That is, the meta-state vector $\mathbf{w}_{k}$ has the expression $\mathbf{w}_{k}=\left[\mathbf{x}_{k} ; \theta_{k}\right]$, with subscription $k$ indicating the time step and used in the subsequent explanation. The parameters in $\theta$ are assumed to be locally time-invariant or slowly varying compared to the process. Accordingly, the augmented system is described by

$$
\begin{aligned}
\mathbf{w}_{k} & =\mathbf{F}\left(\mathbf{w}_{k-1}, u_{k-1}\right) \\
y_{k} & =\mathbf{H} \mathbf{w}_{k}
\end{aligned}
$$

where

$$
\begin{aligned}
\mathbf{F}\left(\mathbf{w}_{k-1}, u_{k-1}\right) & =\left[\begin{array}{c}
\mathbf{f}\left(\mathbf{x}_{k-1}, u_{k-1}\right) \\
\boldsymbol{\theta}_{k-1}
\end{array}\right], \\
\mathbf{H} & =\left[\begin{array}{ll}
\mathbf{C} & \mathbf{0}^{1 \times[l+m \times n]}
\end{array}\right] .
\end{aligned}
$$

The recursive estimation of the state-space model with Kalman filter consists of two phases, prediction and correction. The main equations are given by:
1) prediction phase:

$$
\begin{aligned}
& \hat{\mathbf{w}}_{k}^{-}=\mathbf{F}\left(\hat{\mathbf{w}}_{k-1}, u_{k-1}\right) \\
& \mathbf{P}_{k}^{-}=\mathbf{D}_{k} \mathbf{P}_{k-1} \mathbf{D}_{k}^{T}+\mathbf{Q}_{k-1}
\end{aligned}
$$

2) correction phase:

$$
\begin{aligned}
\mathbf{K}_{k} & =\mathbf{P}_{k}^{-} \mathbf{H}_{k}^{T}\left(\mathbf{H}_{k} \mathbf{P}_{k}^{-} \mathbf{H}_{k}^{T}+\mathbf{R}_{\mathbf{k}}\right)^{-1} \\
\hat{\mathbf{w}}_{k} & =\hat{\mathbf{w}}_{k}^{-}+\mathbf{K}_{k}\left(y_{k}-\mathbf{H}_{k} \hat{\mathbf{w}}_{k}^{-}\right) \\
\mathbf{P}_{k} & =\left(\mathbf{I}-\mathbf{K}_{k} \mathbf{H}_{k}\right) \mathbf{P}_{k}^{-}
\end{aligned}
$$

In the prediction phase, at step $k$, the a priori estimate of the state $\hat{\mathbf{w}}_{k}^{-}$, is given by the a posteriori state at the previous step, $\hat{\mathbf{w}}_{k-1}$, as shown in (11). The estimate error covariance $\mathbf{P}_{k}$ is propagated according to (12), wherein $\mathbf{Q}_{k}$ is a diagonal matrix containing the process noise covariance, and $\mathbf{D}_{k}$ is the process Jacobian with respect to the variables involved, with each element $D_{[i, j]}$ computed by:

$$
D_{[i, j]}=\frac{\partial F_{[i]}}{\partial w_{[j]}}\left(\hat{\mathbf{w}}_{k-1}, u_{k-1}\right)
$$

In the correction phase, $\mathbf{K}_{k}$ in (13) is called Kalman filter gain, wherein $\mathbf{R}_{k}$ is a scalar measurement noise covariance. The updated state is computed in (14), and the updated estimate error covariance is given by (15).

Although the Kalman filter is an effective way of estimating the state and parameters of a discrete-time controlled process, its performance in estimating the time-varying parameters is degraded by the fact that it refers to the entire history of past measurements [29]. This is particularly troublesome since the activity of stimulated muscles may vary, with prolonged or repetitive stimulation leading to different muscle states. In order to track the time-varying muscle condition, a forgetting factor $\lambda$ is deliberately introduced as proposed in [30]. Consequently, equation (12) and (13) can be rewritten as

$$
\begin{aligned}
\mathbf{P}_{k}^{-} & =\mathbf{D}_{k} \mathbf{P}_{k-1} \mathbf{D}_{k}^{T} / \lambda \\
\mathbf{K}_{k} & =\mathbf{P}_{k}^{-} \mathbf{H}_{k}^{T}\left(\mathbf{H}_{k} \mathbf{P}_{k}^{-} \mathbf{H}_{k}^{T}+\lambda\right)^{-1}
\end{aligned}
$$

Choosing forgetting factor $\lambda \in[0,1]$ depends on how much we hope the filter to forget the past measurements. The forgetting factor is closer to 1, the filter will forget fewer past measurements. A tradeoff between the smoothness of tracking and lag in detecting the changes in model parameters should be considered when forgetting factor is introduced to a Kalman filter. Usually $\lambda \in[0.9,1]$ is suitable for most application.

\section{Results}

\section{A. Muscle Fatigue and Recovery Process}

The data from fatigue-recovery tests were used to observe the relationship between torque and MAV of eEMG during intermittent stimulation and recovery. The data during stimulation plateau was used for analysis. The data were firstly treated as the processing step (1) (2) described in section II. C. Then the average torque, MAV and standard deviations were computed simultaneously. The results are depicted in Fig. 6. The torque of ankle plantar-flexion gradually declined after each fatigue-inducing sequence, until around $90 \%$ of initial torque in all subjects. After 5 minutes rest, the average torque 
recovered less than $5 \%$ and then remained at the same level in all subjects. Except in subject S1, the torque recovered after 10 minutes rest. As a whole, torque transition in fatigue generally showed a similar tendency in all subjects. The MAV of eEMG represented different transitions among these subjects as depicted in Fig. 6 (b). Although the same tendency can be found in subjects S3 and S5, different tendencies are found among the five subjects. The results in S3, S5 show a simple decline of MAV due to fatigue, $\mathrm{S} 4$ shows potentiation phenomenon, while $\mathrm{S} 1, \mathrm{~S} 2$ represent somewhat combined characteristics of potentiation and fatigue. Here, we do not focus on the understanding of the different characteristics. However, we were able to confirm that the eEMG-to-torque relationship was not constant as most prior works assumed, but gradually varying, in this intermittent fatigue protocol.

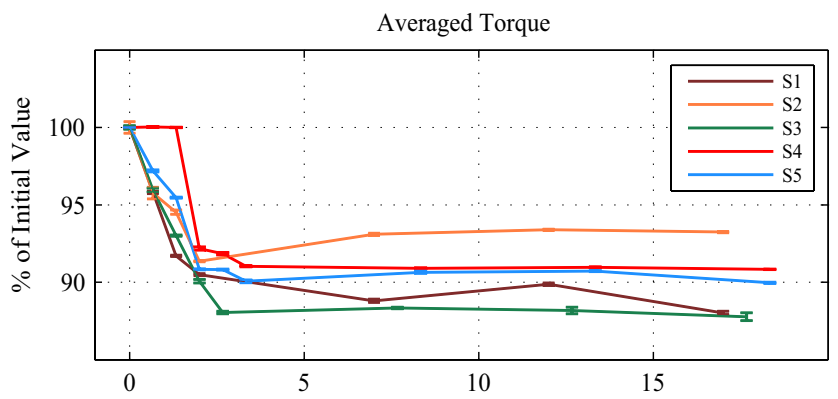

(a)

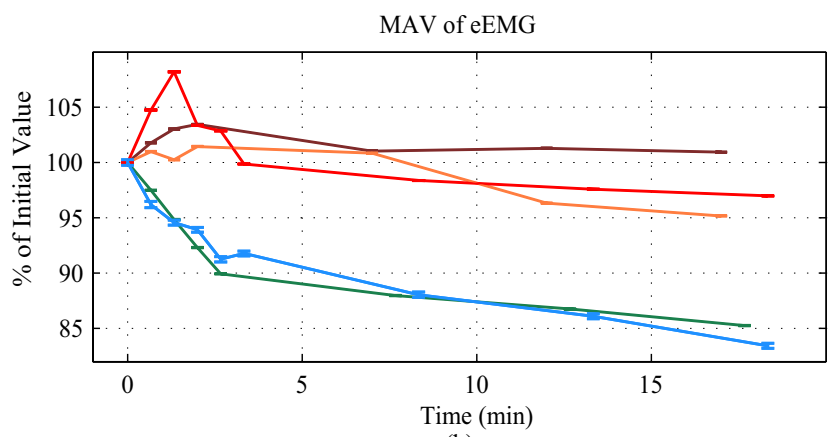

(b)

Fig. 6. (a) Average torque and (b) MAV of eEMG in all subjects during the fatigue-recovery process. The results were represented as mean \pm standard deviation.

\section{B. Model Identification and Validation Using the Ordinary Least Squares (OLS) Method}

The torque was predicted based on past measured torque and past predicted torque as described in section III. A, using random test data. OLS method was performed for model identification. Fig. 7 illustrates the prediction result in subject S5. The data during 97s of random stimulation were separated into two parts at 68s. The model parameters in (4) were identified using the data before 68s. Then torque prediction was calculated using two different methods, as expressed in (5) and (6) respectively, with the data before and after $68 \mathrm{~s}$. It means that the torque prediction after $68 \mathrm{~s}$ is only driven by eEMG information. The corresponding prediction errors, root-mean-square (RMS) error, are also shown in this figure. From the result of the random stimulation test, we can confirm the feasibility of model identification and its prediction performance. For the next step, we need to investigate this in different muscle fatigue states to verify the model predictability.

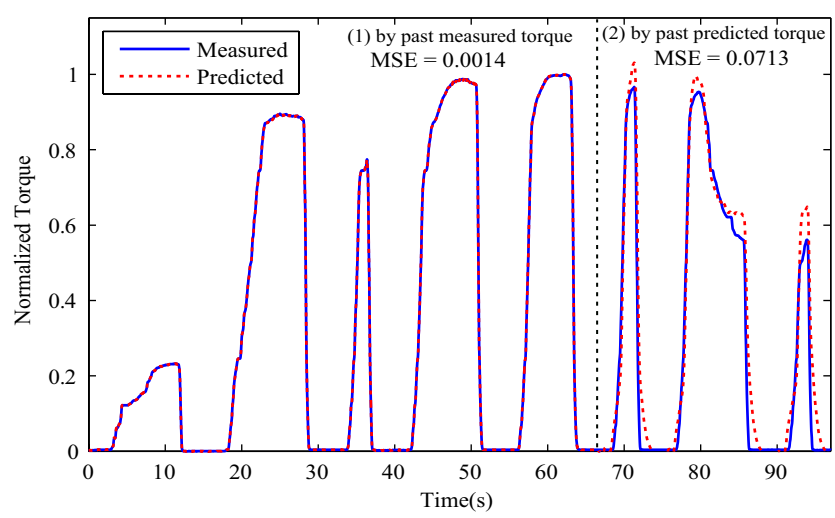

Fig. 7. The measured and predicted torque obtained by eEMG-to-torque model in random test in subject S5. The vertical dotted line is shown to separate the data into two parts. The model was identified by OLS method using the data in the left part. (1) The torque was predicted based on the past measured MAV of eEMG and past measured torque. (2) The torque was predicted based on the past measured MAV of eEMG and past predicted torque, where the prediction was calculated only by eEMG.

The torque prediction was conducted using the OLS method with the fatigue-inducing data in 5 subjects. Two different models, the fixed-parameter model and the adapted-parameter model, were tested. The fixed-parameter model can be explained as follows: the model parameters were identified with the data of the first sequence (fatigue1), and were then crossvalidated for all the remaining sequences (fatigue2-fatigue5). The prediction errors are shown in TABLE II. We found that with the fixed-parameter model, the prediction error became higher and higher as the muscle was more and more fatigued. We then supposed that if the model was identified again with the data in the latest sequence, the prediction could be improved. Therefore, the second method, the adaptedparameter model, was proposed. In this approach, the latest data were used to re-identify the model. That is, the torque prediction of fatigue 3 was based on the model parameters obtained from fatigue2, and so on. The prediction errors with the adapted-parameter model in all subjects are compared in TABLE II. For example, for fatigue3 of subject S3, the RMS error with the adapted model is 0.0345 , as compared to 0.0639 with the fixed model. The average prediction of the adapted model was superior by $16.7-50.8 \%$ compared to the fixed model in all subjects.

In Fig. 8, the predictions with the two methods (fixed and adapted model) and in different fatigue conditions are shown in subject S3. Fatigue3 was not plotted in this figure, as there was only a small difference between fatigue 3 and fatigue 4 . Obviously, FES-generated torque declined with the same stimulation as a result of muscle fatigue. The fixed model could be still used for torque prediction. However, in comparison with fatigue 1 and fatigue 2 , the prediction of fatigue 4 became less precise based on the fixed model, whereas the adapted model contributed to improve torque prediction accuracy, as the 
TABLE II

SUMMARY OF THE PREDICTION ERROR OBTAINED WITH FIXED OR ADAPTED EEMG-TO-TORQUE MODEL

\begin{tabular}{c|c|c|c|c|c|c}
\hline \multirow{2}{*}{ Subject } & \multirow{2}{*}{ Model } & \multicolumn{5}{|c}{ Average RMS Error } \\
\cline { 3 - 7 } & & F1 & F2 & F3 & F4 & F5 \\
\hline \multirow{2}{*}{ S1 } & Fixed & 0.0381 & 0.1009 & 0.2037 & $/$ & $/$ \\
\cline { 2 - 7 } & Adapted & $/$ & $/$ & 0.1272 & $/$ & $/$ \\
\hline \multirow{2}{*}{ S2 } & Fixed & 0.0642 & 0.0767 & 0.1559 & $/$ & $/$ \\
\cline { 2 - 7 } & Adapted & $/$ & $/$ & 0.1298 & $/$ & $/$ \\
\hline \multirow{2}{*}{ S3 } & Fixed & 0.0253 & 0.0467 & 0.0639 & 0.0572 & $/$ \\
\cline { 2 - 7 } & Adapted & $/$ & $/$ & 0.0345 & 0.0254 & $/$ \\
\hline \multirow{2}{*}{ S4 } & Fixed & 0.0511 & 0.0520 & 0.0575 & 0.0702 & 0.0631 \\
\cline { 2 - 7 } & Adapted & $/$ & $/$ & 0.0480 & 0.0447 & 0.0438 \\
\hline \multirow{2}{*}{ S5 } & Fixed & 0.0594 & 0.0641 & 0.0591 & 0.0597 & 0.0715 \\
\cline { 2 - 7 } & Adapted & $/$ & $/$ & 0.0457 & 0.0447 & 0.0476 \\
\hline
\end{tabular}

* F1-F5 respectively denotes the sequence fatigue1-fatigue5 in fatigueinducing test session.

dashdotted black line shown. Therefore, we can conclude that the muscle model parameters are time-varying and gradually change with the effect of fatigue, suggesting that online model estimation can improve torque prediction in fatiguing muscles. This finding motivate us to apply a Kalman filter for online estimation in order to track muscle fatigue.

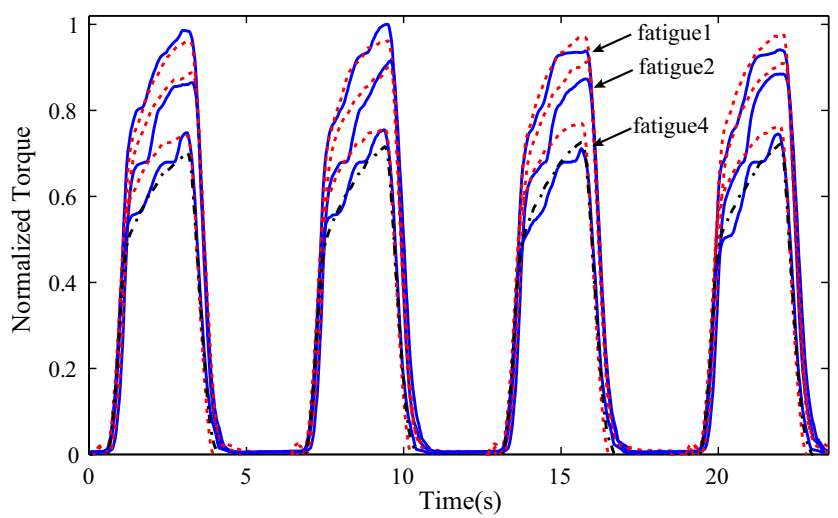

Fig. 8. The measured and predicted torque obtained by eEMG-to-torque model in fatigue-inducing protocol in subject S3. The solid blue lines indicate the measured torques. The dotted red lines represent the corresponding predicted torques based on fixed model which was identified using the data of fatigue 1 . The dashdotted black lines represent the torque prediction of fatigue 4 based on adapted model which was identified using the data of fatigue3. All the torques were normalized by the maximum measured torque in fatigue1.

\section{Validation of Time-Varying Model Estimation with Kalman Filter}

In this part, the time-varying parameter estimation performed by the Kalman filter was evaluated in simulations and with experimental data. The outlines of the muscle model and Kalman filter were described previously. For a PHM $(l, m, n)$ model, we need to estimate the $r=\max (l, m)+(l+m \times n)$ dimensional meta-state. The $\max (l, m)$ parameters relate to the internal states, the rest relate to the past torque and the past MAV of eEMG. The elements of the meta-state vector were initialized as $\hat{w}_{i}(0)=0, i=1,2 \cdots, r$. The initial output estimate $\hat{y}_{0}$ was set at zero. The estimate error covariance was initialized as $\mathbf{P}_{0}=\mathbf{I}$, where $\mathbf{I}$ is an identity matrix.
1) Time-Variant Parameter Tracking in Simulation: In simulation, invariant parameter tracking was evaluated first with the Kalman filter to investigate the stability of the muscle model. Secondly, in order to investigate the filter's robustness to the time-varying fatigue phenomenon, we slowly changed the model parameters at different instants to imitate changes in muscle condition. The advantage of simulation is that the true parameters are known to be compared with the estimated ones. The simulation model order was chosen as $l=2, m=2, n=$ 1 to reduce model complexity, as it is difficult to know how the model output changes when too many parameters change. Thus, four parameters, $a_{1}, a_{2}, \mu_{11}, \mu_{21}$, were estimated via the Kalman filter algorithm in simulation. At the beginning, all the parameters were kept constant. After 33s or 50s, they were changed linearly $\left(a_{1}\right.$ and $\left.\mu_{21}\right)$ or in steps $\left(a_{2}\right.$ and $\left.\mu_{11}\right)$. The pseudorandom binary sequence (PRBS), which is commonly used in muscle identification [25], was chosen as model input. Model input, output and the a posteriori estimate of the output are shown in Fig. 9.
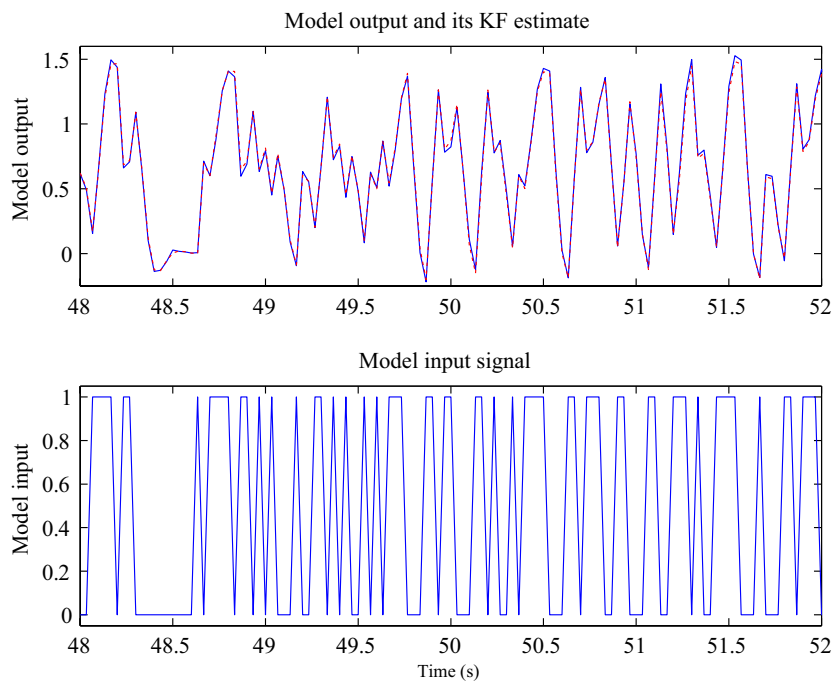

Fig. 9. Data set for model identification. Data were generated by simulation using a PHM $(2,2,1)$. A Kalman filter with forgetting factor 0.97 was used to identify the model.

The corresponding parameter estimates of the model are depicted in Fig. 10. The solid lines indicate the true parameters, while the dotted lines indicate parameter estimates. All the parameters converge steadily after $5 \mathrm{~s}$ when the parameters are static. After $33 \mathrm{~s}$ or $50 \mathrm{~s}$, the model parameters gradually vary, and the estimates track the changes well, which implies that the estimation method is suitable for time-variant parameter tracking with a PHM model.

2) Fatigue Tracking Based on Experimental Data: In the model identification described in section V. B, the adaptedparameter model was proved to be able to improve torque prediction, but identification was performed without automatic tracking function. In this section, the identification and validation of time-varying parameters are considered and performed automatically using the Kalman filter. The model order is chosen at $(3,4,3)$ as described above. The data from successive series of fatigue-inducing tests were concatenated 


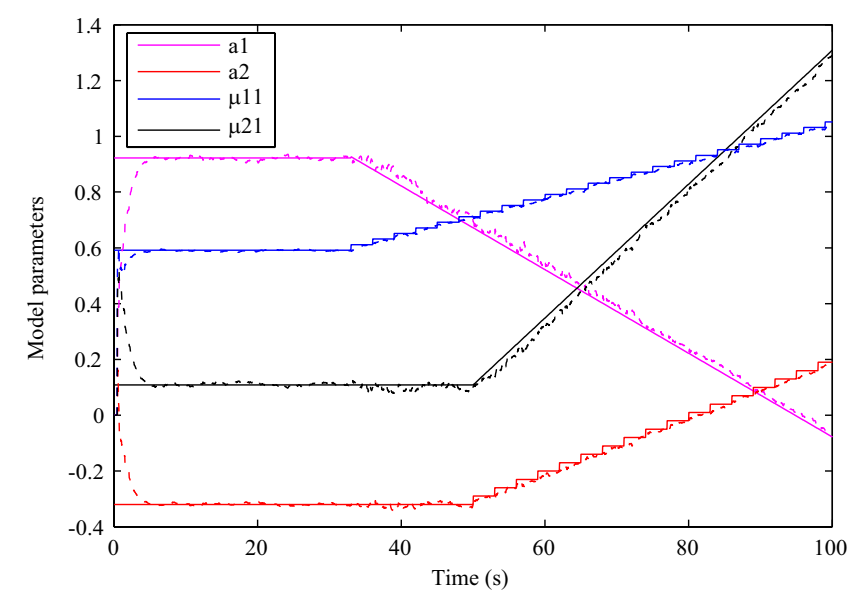

Fig. 10. Convergence and tracking of both static and time-variant parameters. Solid lines indicate true parameters. Dotted lines indicate parameter estimates. All the initial estimates were set at zero.

for estimation. During 100s of intermittent stimulation, the torque of each subject decreased to a different level, because of different muscle fatigue level. The time-varying relationship between MAV of eEMG and torque was revealed in Fig. 6.

Fatigue Dynamics: The estimation of the muscle contraction process can be used to explore the dynamics of fatigue phenomena. The PHM $(3,4,3)$ of the contraction dynamics was fitted to the measured torque collected in the fatigueinducing test session. A Kalman filter with forgetting factor $(\lambda=0.997)$ was used for model estimation. The locations of these poles in subject S1 and S3 are indicated in Fig. 11. The unit circles are also plotted in this figure. All the poles are located within the unit circles, which is of significance in ensuring the model stability under our stimulation protocol. The arrows denote the direction of movement of the z-plane poles. The time-varying property of the poles may also interpret the resulting time-varying model parameters. Moreover, the locations and movements of the z-plane poles in all subjects present similar characteristics, suggesting that it is possible to assess muscle fatigue dynamics from such information. In general, the damping ratio increases when the muscle is highly fatigued. This matches our intuition concerning the effect of muscle fatigue.

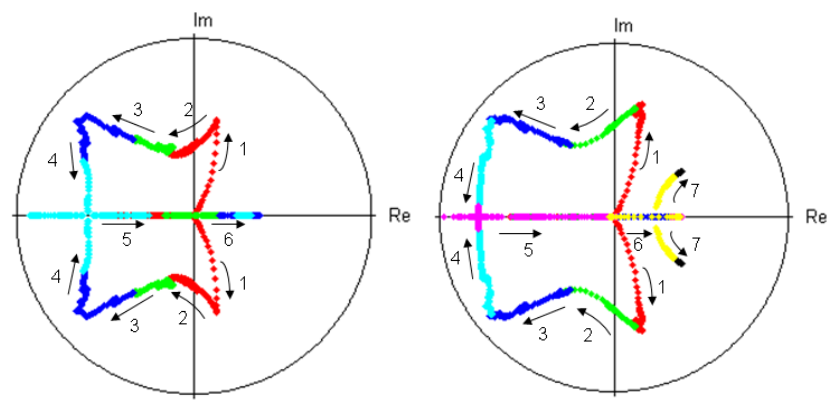

Fig. 11. The changes in the contraction dynamics of muscle behavior due to fatigue during intermittent stimulation in subjects S1 (left) and S3 (right). The arrows denote the direction of "movement" of the z-plane pole. The plot color was changed every 16 s to show the time transition.

Torque Prediction Performance: To investigate the torque

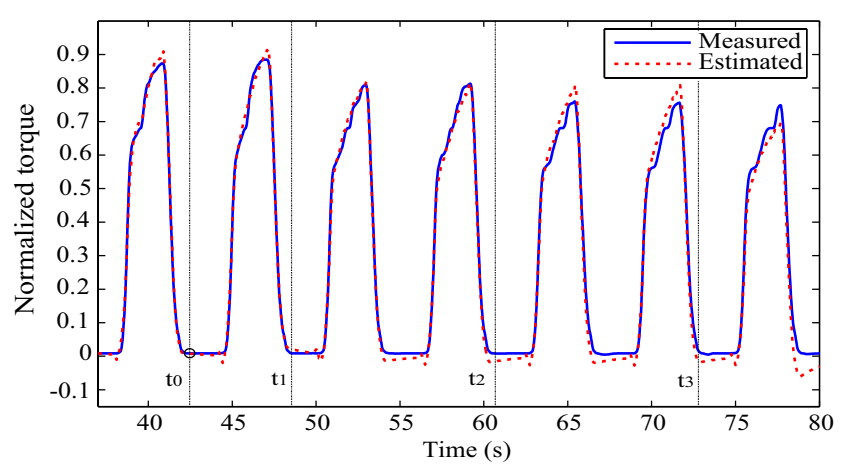

Fig. 12. Measured and estimated torque at time instant $t 0$. For evaluating prediction performance, the prediction errors for prediction horizons $t 1, t 2$, and $t 3(6 \mathrm{~s}, 18 \mathrm{~s}$ and $30 \mathrm{~s}$ respectively) are measured. A Kalman filter with forgetting factor 0.997 was used to estimate the model.

TABLE III

PREDICTION FILTER PERFORMANCE WITH EXPERIMENTAL DATA

\begin{tabular}{c|c|c|c|c}
\hline \multirow{2}{*}{ Subject } & \multirow{2}{*}{ Average error } & \multicolumn{3}{|c}{ Prediction horizon } \\
\cline { 3 - 5 } & & $6 \mathrm{~s}$ & $18 \mathrm{~s}$ & $30 \mathrm{~s}$ \\
\hline \multirow{2}{*}{ S1 } & RMS & 0.0638 & 0.0974 & 0.1282 \\
\cline { 2 - 5 } & Peak & 0.1616 & 0.2990 & 0.3414 \\
\hline \multirow{2}{*}{ S2 } & RMS & 0.0763 & 0.0925 & 0.1110 \\
\cline { 2 - 5 } & Peak & 0.2466 & 0.3402 & 0.4230 \\
\hline \multirow{2}{*}{ S3 } & RMS & 0.0278 & 0.0314 & 0.0366 \\
\cline { 2 - 5 } & Peak & 0.0743 & 0.0962 & 0.1146 \\
\hline \multirow{2}{*}{ S4 } & RMS & 0.0524 & 0.0534 & 0.0556 \\
\cline { 2 - 5 } & Peak & 0.1208 & 0.1523 & 0.1697 \\
\hline \multirow{2}{*}{ S5 } & RMS & 0.0387 & 0.0418 & 0.0437 \\
\cline { 2 - 5 } & Peak & 0.1036 & 0.1360 & 0.1510 \\
\hline
\end{tabular}

prediction performance in time, different prediction horizons, $6 \mathrm{~s}, 18 \mathrm{~s}$ and $30 \mathrm{~s}$, were tested. The idea is illustrated in Fig. 12 , which features the estimated model at time instant $t 0$. The torque predictions were computed using (4) and only driven by the eEMG, considering a static system within the prediction horizon. At every torque sample for a given prediction horizon, the RMS error and peak error were evaluated. The prediction errors in all subjects are quantified in Table III. Eighteen seconds was considered to be an appropriate prediction horizon, as it provided a tradeoff between sufficient interval for measurement update in Kalman filter and satisfactory prediction performance. The prediction errors for the $18 \mathrm{~s}$ prediction horizon in subject S3 are plotted in Fig. 13. The solid blue line indicates RMS error, while the dotted black line indicates peak error.

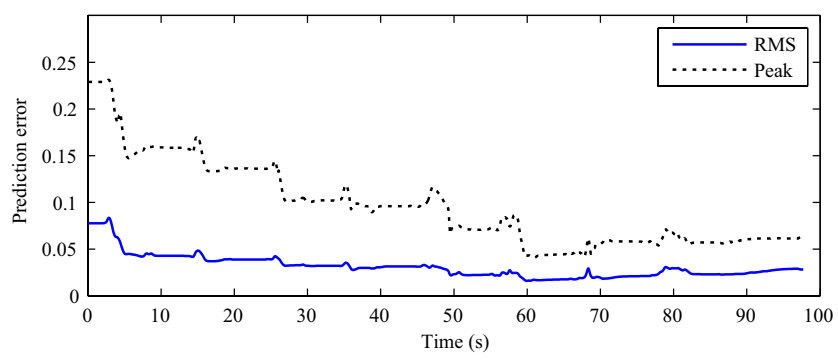

Fig. 13. RMS and peak prediction error of 18 s prediction horizon in $\mathrm{S} 3$.

Predictive Performance in Sensing Failure: In order to 
evaluate tracking robustness in the event of interruption in recording or failure, torque prediction was integrated into the fatigue tracking task. Any event such as a missing torque measurement, was assumed to result in tracking failure. Fig. 14 reveals fatigue tracking performance in subject S3 when muscle torque declined to $76 \%$ of the maximum torque during the 100s-stimulation. Assuming measurement was not available from time instant $t 0$ to $t 1$, the estimated model at $t 0$ was used to predict torque until instant $t 1$. When prediction was executed, the model was only driven by the eEMG, while the online model estimation was suspended. To evaluate prediction performance in different muscle fatigue states, this process was repeated until the end of stimulation, where the measurement update in Kalman filter was switched off for 18s. The prediction tracks the measured torque well, suggesting that if torque measurement is unavailable or unreliable, the predicted torque based on eEMG can be used to bridge such gaps for the prolonged application of FES.

Some of the estimated parameters are also depicted in Fig. 14. The middle plot shows the parameters of the past torque, while the parameters of the 3rd power of the past MAV are shown in the lower plot. Although the parameters gradually vary, representing varying myoelectrical mechanical correlation during muscle fatigue, the proposed muscle model and Kalman filter algorithm with forgetting factor provide good prediction performance, as shown in the upper plot.

\section{DISCUSSION}

Stimulus-evoked EMG has been previously proposed for the prediction of the mechanical behavior of muscles in FES rehabilitation systems [18] [19]. These prediction methods were investigated on the hypothesis of a fixed eEMGto-torque model during ES. Some other researchers found that this relationship was time-variant under different muscle conditions [20] [21]. However, they have not proposed a feasible, effective method for torque prediction. The timevarying property of myoelectrical and mechanical muscle behavior is shown in Fig. 6. It implies the limitations of the torque prediction method based on the fixed eEMG-to-torque model and increases the difficulties of torque estimation. The present work proposes the use of the Hammerstein structure to represent muscle contraction dynamics with MAV of eEMG as input, where model estimation is performed by a Kalman filter for fatigue tracking.

The prediction result presented in Fig. 12 reveals good performance of the predictive filter, successively acquiring the mechanical behavior of the muscle under FES. In addition, the error values given in Table III indicate that neither RMS error nor peak error increases significantly when the prediction horizon is extended. It indicates that the proposed eEMGto-torque model properly fits muscle behavior. If prediction quality diminishes when an expanded prediction horizon is selected, this is probably due to the variations of muscle fatigue levels during the horizon. This can occur when prolonged or repetitive stimulation is delivered to the muscle. In this application, $18 \mathrm{~s}$ prediction horizon is considered as the optimal choice.
The muscle contraction dynamics model has been described in a previous work [19], in continuous stimulation, with the recursive least squares method used for identification. However, there was no significant consideration of different fatigue conditions in intermittent stimulation, as illustrated in Fig. 14. In this study, the proposed estimation method was validated for ankle torque prediction and fatigue tracking using eEMG under isometric condition, it would be promising to verify it in dynamic conditions by introducing a torque-joint angle function to the proposed muscle model as in [25].

In this work, we did not consider the effect of day-to-day changes, but as the online identification is able to identify parameters for different subjects, which implies that this method itself can work even if there are day-to-day changes if we do not care about how the parameters change depending on the experimental set-up, for example, electrode position. Of course, it is important to investigate the effect of different experimental set-ups. In this case, we may introduce a tuning function to offset the day-to-day variances.

\section{CONCLUSION}

The objective of this work is to develop a torque estimation method which can be used to track FES-induced muscle fatigue. In this work, we confirmed that muscle contraction model parameters were time-varying during intermittent stimulation in five SCI subjects. An estimation method of FESinduced torque based on eEMG signals was evaluated in its prediction performance. A time-varying eEMG-to-torque model was employed to represent the myoelectrical and mechanical behavior of stimulated muscles, where the model parameters were estimated by a Kalman filter with forgetting factor. The results of the proposed method for the fatigue tracking task represent a feasible and effective torque prediction performance in isometric condition in all subjects. In terms of improvements in fatigue tracking, when the measurement of torque sensor suffers from recording interruption, the proposed method can bridge these problems and provide sufficiently accurate fatigue tracking only on eEMG measurement. Therefore, we conclude that the proposed estimation method can contribute to precise torque prediction in presence of muscle fatigue. The predicted torque can be further used for adaptive closed-loop FES control, considering the compensation of muscle fatigue. Future work will be extended to adaptive FES control for muscle fatigue compensation in SCI patients. We will also endeavor to further improve the identification by means of multiple eEMG measurements and more selective stimulation. By introducing a joint dynamics model and joint angle sensors along with the proposed method, we will work on FES close-loop control based on eEMG sensing without the use of torque sensors.

\section{ACKNOWLEDGMENT}

This work is partly supported by China Scholarship Council (CSC) for the first author's PhD study. The authors would like to thank Dr. Charles Fattal and Dr. Maria Papaiordanidou for their helps during the experiments at Propara Rehabilitation Center. 

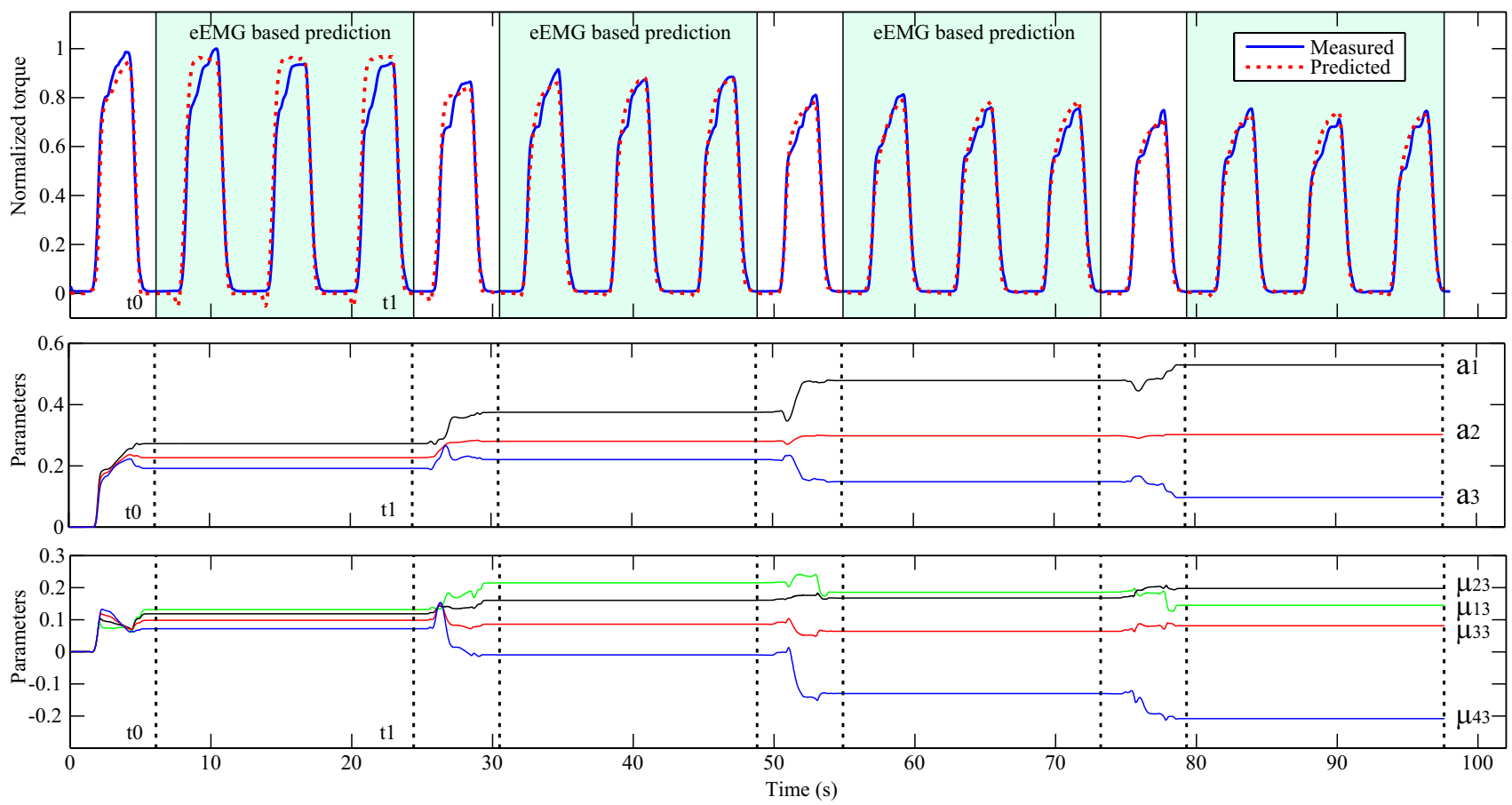

Fig. 14. Fatigue tracking based on eEMG-to-torque model and Kalman filter in subject $\mathrm{S} 3$. The model estimated at $t 0$ was used to predict the torque from $t 0$ to $t 1$, while the online estimation was switched off for $18 \mathrm{~s}$ (with green background color in upper plot). This process was repeated until the end of stimulation. Upper: The solid blue line indicates the measured torque, while the dotted red line indicates the predicted torque. Middle: The estimated model parameters of the past torque. Lower: The estimated model parameters of the 3rd power of the past MAV.

\section{REFERENCES}

[1] R. J. Triolo and K. Bogie, "Lower Extremity Applications of Functional Neuromuscular Stimulation After Spinal Cord Injury", Topics in Spinal Cord Injury Rehabilitation, vol. 5, no. 1, pp. 44-65, 1999.

[2] S. Mangold, T. Keller1, A. Curt and V. Dietz, "Transcutaneous Functional Electrical Stimulation for Grasping in Subjects with Cervical Spinal Cord Injury", Spinal Cord, vol. 43, pp. 1-13, 2005.

[3] C. A. Pelletier, and A. L. Hicks, "Muscle Fatigue Characteristics in Paralyzed Muscle after Spinal Cord Injury", Spinal Cord, vol. 49, pp. 125-130, 2011.

[4] S. A. BInder-Macleo and L. Snyder-Mackler, "Muscle Fatigue: Clinical Implications for Fatigue Assessment and Neuromuscular Electrical Stimulation", Physical Therapy, vol. 73, no. 12, pp. 902-910, 1993.

[5] L. W. Chou and S. A. Binder-Macleod, "The Effects of Stimulation Frequency and Fatigue on the Force-Intensity Relationship for Human Skeletal Muscle", Clinical Neurophysiology, vol. 118, pp. 1387-1396, 2007.

[6] Z. Z. Karu, W. K. Durfee, and A. M. Barzilai, "Reducing Muscle Fatigue in FES Applications by Stimulating with N-Let Pulse Trains", IEEE Transactions on Biomedical Engineering, vol. 42, no. 8, pp. 809-817, 1995.

[7] Y. Shimada, H. Ito, et al., "Reduction of Muscle Fatigue by CatchlikeInducing Intermittent Electrical Stimulation in Rat Skeletal Muscle", Biomedical Research, vol. 27, no. 4, pp. 183-189, 2006.

[8] G. M. Graham, T. A. Thrasher, and M. R. Popovic, "The Effect of Random Modulation of Functional Electrical Stimulation Parameters on Muscle Fatigue", IEEE Transactions on Neural Systems and Rehabilitation Engineering, vol. 14, no. 1, pp. 38-45, 2006.

[9] T. Kesar and S. Binder-Macleod, "Effect of Frequency and Pulse Duration on Human Muscle Fatigue During Repetitive Electrical Stimulation", Experimental Physiology, vol. 91, no. 6, pp. 967-976, 2006.

[10] D. W. Russ, K. Vandenborne, and S. A. Binder-Macleod, "Factors in Fatigue During Intermittent Electrical Stimulation of Human Skeletal Muscle", Journal of Applied Physiology, vol. 93, pp. 469-478, 2002.

[11] J. A. Hoffer, "Closed-Loop, Implanted-Sensor, Functional Electrical Stimulation System for Partial Restoration of Motor Functions", United Stated Patent 4750499 , vol. 19, 1988.

[12] R. Riener, J. Quintern and G. Schmidt, "Biomechanical Model of the Human Knee Evaluated by Neuromuscular Stimulation", Journal of Biomechanics, vol. 29, no. 9, pp. 1157-1167, 1996.

[13] J. Mizrahi, D. Seelenfreund, et al., "Predicted and Measured Muscle Forces After Recoveries of Differing Durations Following Fatigue in Functional Electrical Stimulation", Artificial Organs, vol. 21, no. 3, pp. 236-239, 1997.

[14] J. Ding, A. S. Wexler, and S. A. Binder-Macleod, "A Predictive Model of Fatigue in Human Skeletal Muscles", Journal of Applied Physiology, vol. 89 , no. 4 , pp. 1322-1332, 2000.

[15] N. C. Chesler and W. K. Durfee, "Surface EMG as a Fatigue Indicator During FES-induced Isometric Muscle Contractions", Journal of Electromyography and Kinesiology, vol. 7, no. 1, pp. 27-37, 1997.

[16] A. Erfanian, H. J. Chizeck, and R. M. Hashemi, "Excitation-Contraction Fatigue During Sustained Electrical Stimulation of Paralyzed Muscle", in 18th Annual International Conference of the IEEE Engineering in Medicine and Biology Society, Amsterdam, Netherlands, pp. 1460-1461, 1996.

[17] J. Mizrahi, M. levy, H. Ring, E. Isakov, and A. Liberson, "EMG as an indicator of fatigue in isometrically FES-activated paralyzed muscles", IEEE Transactions on Neural Systems and Rehabilitation Engineering, vol. 2, no. 2, pp. 57-65, 1994.

[18] D. Tepavac and L. Schwirtlich, "Detection and Prediction of FESInduced Fatigue", Journal of Electromyography and Kinesiology, vol. 7, no. 1 , pp. 39-50, 1997.

[19] A. Erfanian, H. J. Chizeck, and R. M. Hashemi, "Using Evoked EMG as a Synthetic Force Sensor of Isometric Electrically Stimulated Muscle", IEEE Transactions on Biomedical Engineering, vol. 45, no. 2, pp. 188202, 1998.

[20] J. Mizrahi, E. Isakov and Z. Suzak, "Myoelectric and Force Characteristics in Transcutanteous Isometric FES", Basic and Applied Myology, vol. 4, no. 2, pp. 147-154, 1994.

[21] N. Y. Yu and S. H. Chang, "Mechanical and Electromyographic Response to Stimulated Contractions in Paralyzed Tibialis Anterior Post Fatiguing Stimulations", in 13th International Conference on Biomedical Engineering, Singapore, pp. 1667-1671, 2009.

[22] C. Frigo, M. Ferrarin, W. Frasson, E. Pavan and R. Thorsen, "EMG Signals Detection and Processing for On-Line Control of Functional Electrical Stimulation", Journal of Electromyography and Kinesiology, vol. 10, pp. 351-360, 2000.

[23] E. J. Dempsey and D. T. Westwick, "Identification of Hammerstein Mod- 
els With Cubic Spline Nonlinearities", IEEE Transaction on Biomedical Engineering, vol. 51, no. 2, pp. 237-245, 2004.

[24] T. L. Chia, P. Chow and H. J. Chizeck, "Recursive Parameter Identification of Constrained Systems: An Applicaiton to Electrically Stimulated Muscle", IEEE Transaction on Biomedical Engineering, vol. 38, no. 5, pp. 429-442, 1991.

[25] W. Farahat and H. Herr, "A Method Identification of Electrically Stimulated Muscle", in 27th Annual Conference of the IEEE Engineering in Medicine and Biology Society, Shanghai, China, pp. 6225-6228, 2005.

[26] L. A. Bernotas, P. E. Crago, and H.J. Chizeck, "A Discrete-Time Model of Electrically Stimulated Muscle", IEEE Transaction on Biomedical Engineering, vol. BME-33, no. 9, pp. 829-838, 1986.

[27] J. Rissanen, "Modeling by Shortest Data Description", Automatica, vol. 14, pp. 465 C 471, 1978.

[28] J. Rissanen, "A Universal Prior for Integers and Estimation by Minimum Description Length", The Annals of Statistics, vol. 11, no. 2, pp. 416 C 431, 1983.

[29] G. A. Mack and V. K. Jain, "Speech Parameter Estimation by Timeweighted-Error Kalman Filter", IEEE Transaction on Acoustics, Speech and Signal Processing, vol. ASSP-31, no. 5, pp. 1300-1303, 1983.

[30] D. Y. Feng, L. Y. Min, et al., "Unscented Kalman Filter for Time Varying Spectral Analysis of Earthquake Ground Motions", Applied Mathematical Modeling, vol. 33, pp. 398-412, 2009.

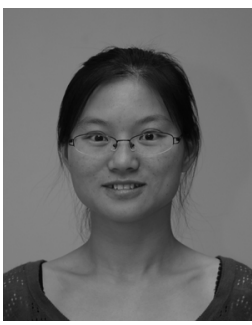

Qin Zhang (S'09) received the B.S. degree in mechanical engineering in 2000, and the M.S. at Huazhong University of Science and Technology in 2003. She was a lecturer in School of Computer Science and Engineering, Wuhan Institute of Technology from 2003. She is currently a $\mathrm{PhD}$ student at DEMAR project, LIRMM/INRIA, France. Her research interests include modeling and control of biomechanical system, and the application of myoelectrical signals.

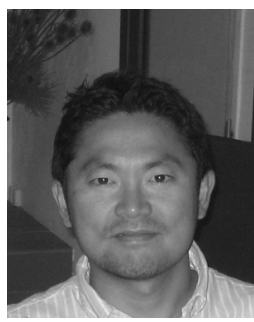

Mitsuhiro Hayashibe (M'04) received the B.S. degree in mechano-aerospace engineering from Tokyo Institute of Technology in 1999. He obtained the M.S. and Ph.D. from University of Tokyo, graduate school of engineering in 2001 and 2005. He was an assistant professor at Jikei University School of Medicine, Department of Medicine from 2001 to 2006. He was a postdoctoral fellow at INRIA Sophia Antipolis and LIRMM of CNRS/University of Montpellier, France in 2007. Since 2008, he is a research scientist with INRIA and LIRMM, Computational Medicine and Neurosciences, DEMAR project. His research interests include modeling and identification of neuromuscular dynamics and computational rehabilitation. He received Best Paper Award from Journal of Japanese Society for Computer-aided Surgery and CAS Young Investigator Award, Gold Prize from Hitachi Medical Systems. He is a member of Engineering in Medicine and Biology Society, IEEE.

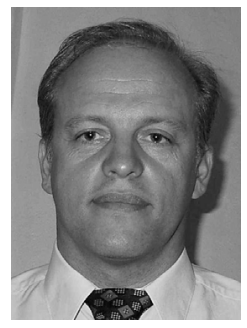

Philippe Fraisse (M'03) received the M.S. degree in electrical engineering from Ecole Normale Superieure de Cachan in 1988. He received the $\mathrm{Ph} . \mathrm{D}$. degree in Automatic Control in 1994. He is currently Professor at the University of Montpellier, France. He is the head of robotics department (LIRMM) and co-chair of French National Workgroup (GDR Robotique) working on Humanoid Robotics (GT7). He is also a member of JRL-France scientific board (Japanese-French joint Laboratory for Robotics, AIST-JRL) and a member of IEEE. His research interests include modeling and control applied to robotic and rehabilitation fields, including humanoid robotics, robotics for rehabilitation.

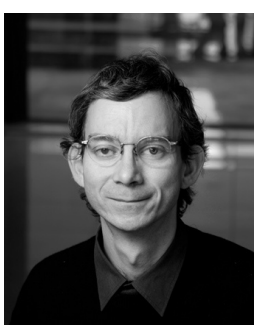

David Guiraud (M'09) obtained the M.S. degree at Ecole Centrale de Paris in 1990, the Ph.D. in biomedical engineering in 1993. He founded DEMAR team at INRIA Sophia-Antipolis Mediterranee and LIRMM in 2004 after being involved in different European Projects at the school of Medicine of Montpellier and INSERM lab. He obtained the bronze medal from CNRS in 2005 in the Communication and Information Technology section. Now he is senior researcher at INRIA. He is a life member of IFESS (International Functional Electrical Stimulation Society) and member of IEEE EMB society. He obtained an award from french academie des sciences in 2010 about the fruitful collaboration between information technology, medicine and and transfer towards industry and clinics. 